zurïckgeblieben. Wir haben auch durch directe Versuche, die schon vọn Muskulus und Barfoed beobachtete Gährungsfähigkeit des Dextrins nachgewiesen, insbesondere dann, wenn das Dextrin sich in Zuckerlösung befindet. Es ist also wohl mehr als wahrscheinlich, dass die Differenz im Zuckergehalte vor und nach dem Erhitzen der Lösung mit Säure auf Rechnung von Dextrin zu beziehen ist. Wäre diess durch absoluten Alcohol gefällt worden, oder hätten sie die Lösung dialysirt, dann wïrden Ch. und L. dieselbe Erfahrung gemacht, welche 0 . Nasse ${ }^{1}$ ) und wir gemacht haben, dass die Erhitzang mit Säure das Reductionsvermögen der Zuckerlösung nicht zu ändern im Stande ist, dass also kein Grund vorliegt, den Leberzucker als ein Gemenge von Maltose und Dextrose anzusehen.

\title{
Ueber Sehstörungen nach Operationen im Bereich des Vorderhirns.
}

Von

\section{Prof Sigm. Exmer und Dr. Josef Paneth.}

Die Frage, ob blos Exstirpationen im Bereich der von $\mathrm{Munk}$ abgegrenzten Sehsphäre, oder auch an anderen Theilen des Hundehirns, insbesondere am Vorderhirn, Sehstörungen nach sich ziehen, steht im Vordergrunde der Discussion, die sich an die Localisation der Functionen im Gehirn knuipft. Dieser Umstand, der ausser in der Literatur auch noch in den Verhandlungen der physiologischen Section der diesjährigen Naturforscher-Versammlung zu Tage trat, rechtfertigt es, wenn wir nachstehend iiber unsere einschlägigen Erfahrungen kurz berichten, die wir im Verlaufe einer nach ganz anderen Zielen gerichteten Untersuchung zu machen Gelegenheit hatten.

Wir haben an sechs Hunden deu Gyrus sigmoideus der einen Seite oder theilweise exstirpirt, und in fünf dieser Fälle Sehstörungen beobachtet, welche bis zu vier Wochen anhielten. In keinem war

1) O. Nasse, Bemerkungen zur Physiolopie der Kohlehydrate. Pflügers Archiv. Bd. XIV. 
Ueber Sehstörungen nach Operationen im Bereich des Vorderhirns. 63

durch Obduction irgend eine Abnormität in der hinteren Hälfte der Gehirnconvexität oder ihrer Häute nachweisbar, noch irgend eine Veränderung an der Basis u. dergl,, welche eine Erklärung der Sehstörung abgeben könute. Der einzige Fall, der keine Sẹstörung zeigte, betraf einen recht jungen Hund, dem ausser dem genannten Gyrus noch ein Stück Rinde in der Grösse der Munk'schen Stelle $A_{1}$ und knapp vor derselben gelegen entfernt war.

Zwei weitere Hunde, denen ebenfalls zu anderen Zwecken das Rindenfeld des Facialis einseitig exstirpirt war, zeigten keine Sehstörungen.

Ferner beobachteten wir in sechs Versuchen, bei welchen durch anderweitige (seinerseits zu beschreibende) Operation der Gyrus sygmoideus und seine nächste Umgebung (bis zu $5 \mathrm{~mm}$ ) schwer geschädigt wurde, jedesmal Sehstörungen, welche bis zu fünf Wochen anhielten. Auch in diesen Fällen beschränkten sich die makroskopisch nachweisbaren Veränderungen, wie die Obduction zeigte, auf die nächste Umgebung der Operationsstelle.

Dagegen fehlte jede Sebstörung vollständig in zwei zur Controlle ausgeführten Experimenten, in welchen die Operation genau wie in früheren bis zu den Verletzungen der pia mater (inclusive) durchgeführt, dann aber innegehalten wurde, so dass die Rinde selbst intact blieb - zum Beweis, dass die Sehstörungen nicht an irgendwelche durch die Operationen gesetzte nebensächliche Momente, sondern nur an die Verletzung der Rinde geknüpft waren.

Die Hunde, über welche wir hier berichtet haben, waren mit wenigen Ausnahmen bei vollständigem Woblbefinden, abgesehen von den durch die Operation bedingten und allgemein bekannten motorischen Störungen. Die Heilung war in der grössten Mehrzabl der Fälle per primam intentionem erfolgt.

Da Beobachtungen über diese Art der Sebstörung bei diesen Thieren nicht zunächst das Ziel unserer Versuche waren, so haben wir uns begnügt, ihr Vorhandensein, den Zeitpunkt ibres Auftretens und Verschwindens zu constatiren. Wir bedienen uns desshalb des allgemein gefassten Ausdruckes ,gekreuzte Sehstörung“, und meinen damit die bekannte Ersclieinung, dass Rindenoperirte Hunde 
Gegenstände in der (zur Operationsstelle) gekreuzten Sehfeldbälfte nicht oder weniger beachten, als in der gleichseitigen. Unsere Prüfungen bestanden zumeist darin, dass der eine Beobachter das Thier beschäftigte, indem er ihm in der geschlossenen Hand ein Stiuck Fleisch vorbielt. An diesem schniiffelt und leckt der Hund herum, und es vertritt auf diese Weise das Object, das man einen Menschen bebufs einer ähnlichen Untersuchung fixiren lässt. Der andere Beobachter führte wäbrenddem ein Stiick Fleisch, das an einem langen Eisendraht befestigt war, in das Gesichtsfeld des Thieres. Dann verlässt ein normaler Hund nach beiden Seiten, einer mit Sehstörung nach der nicht vernachlässigten Seite hin, die geschlossene Hand, sowie er das Fleischstück am Drahte erbliekt and schnappt nach diesem, während der Hund mit Sehstö. rung später oder erst nach einigem Herumbewegen des Fleisches, oder auch gar nicht sich darum bekümmert. Andere Proben, wie das Erschrecken vor einem Zïndhölzchen, das Blinzeln bei Annäherung des Fingers, ehe derselbe die Cornea berührt, sowie das Ergreifen desjenigen von zwei symmetrisch vorgehaltenen Fleischstiicken, welches vor das Auge der der Operation gleichnamigen Seite gehalten wird, wurden ebenfalls angestellt. Dieses Verhalten war vorhanden bei völlig intacter Beweglichkeit des Kopfes und der Wirbelsäule, kann also schon darum nicht auf Motilitätsstörungen bezogen werden.

Die Sehstörung zeigte sich sowie das Thier in den ersten Tagen nach der Operation untersucht werden konnte. Ihre Intensität war manchmal, aber nur selten, so gross, dass sich in dem Protocoll die Bemerkung findet: Es ist überhaupt nicht auszumachen, ob das Thier auf dem gekreuzten Auge sieht. Sie ging in allen Fällen allmäblich zurïck, und verschwand vollständig bei denjenigen Thieren, die lang genug am Leben erhalten wurden. Die Dauer war verschieden $(7,10,12,16,17,20,24,29,37$ Tage nach der Operation nachweisbar). Wir müssen uns demnach der Ansicht derjenigen Forscher anschliessen, welche nicht ausschliesslich die hinteren Partieen der Convexität der Hemisphären in Beziehung zur Seh-Funetion bringen. Es ist dies die Mehrzahl der Beobachter, unter ihnen Goltz, Hitzig, Luciani und Seppilli, L 0 eb u. A. 